\title{
O LUGAR DE PEIRCE NA HISTÓRIA DA LÓGICA: UM DIÁLOGO COM A TRADIÇÃO FREGE/RUSSELLIANA
}

\author{
Renan H. Baggio \\ Programa de Estudos Pós-graduados em Filosofia - Doutorado \\ Pontifícia Universidade Católica de São Paulo - PUC/SP \\ renanhbaggio@gmail.com \\ bolsista CAPES
}

Resumo: O presente trabalho tem como intuito apresentar os estudos de Charles Sanders Peirce, assim como sua relevância para as investigações acerca da lógica e de sua natureza. Peirce deixou diversas contribuições para o estudo da lógica que parecem ter sido ofuscadas pelos trabalhos de pensadores enaltecidos pela tradição. Diante disso, nossos esforços visam apresentar como Peirce desenvolveu, em certa medida ainda mais cedo, as questões levantadas por Frege, Russell e outros expoentes da lógica, além de ter trabalhado em teorias originais que elevam seus esforços à autenticidade. Para tanto, abordamos na primeira seção as duas vertentes de pensamento as quais compreendem a lógica enquanto linguagem universal e como teoria dos modelos, de modo a inserir a teoria peirciana em meio a essas abordagens. Na segunda seção analisamos alguns aspectos originais do pensamento peirciano e sua relevância para a teoria da lógica. Por fim, na terceira seção apresentaremos os aspectos que permitem Peirce se equiparar aos teóricos consagrados da lógica, sobretudo Frege e Russell.

Palavras-Chave: História da lógica. Lógica de Peirce. Linguagem universal. Lógica e teoria dos modelos.

\section{THE PLACE OF PEIRCE IN THE HISTORY OF LOGIC: A DIALOGUE WITH THE FREGE/RUSSELLIAN TRADITION}

\begin{abstract}
The current work intends to exhibit the studies of Charles Sanders Peirce, as well as its relevance to the investigations about logic and its nature. Peirce left several contributions to the study of logic that seem to have been overshadowed by the work of thinkers praised by tradition. In the light of this, our efforts are aimed at showing how Peirce developed, to some extent earlier, the issues raised by Frege, Russell, and other exponents of logic, as well as working on original theories that elevate their efforts to authenticity. In order to do so, we approach in the first section the two strands of thought which comprise logic as a universal language and as model-theoretical view, in order to insert the Peircian theory in the midst of these approaches. In the second section we analyze some original aspects of Peircian thought and its relevance to the theory of logic. Finally, in the third section we show the aspects that allow Peirce to be equated with the consecrated theoreticians of logic, especially Frege and Russell.
\end{abstract}

Keywords: History of logic. Peirce's logic. Universal language. Logic and model theory. 


\section{Introdução}

De modo a compreender o papel de Charles Sanders Peirce no percurso do pensamento filosófico, sobretudo, seu papel nos estudos acerca da natureza da lógica, é preciso considerar certos aspectos de sua teoria e compará-los com as questões tratadas pelos pensadores enaltecidos pela tradição. Diante dessa análise, buscamos clarificar o lugar de Peirce na história da lógica e estabelecer em que medida seus escritos são tão importantes para a construção do conhecimento humano quanto aqueles deixados por pensadores consagrados. É possível considerar Peirce um lógico equiparável a Aristóteles ou a Frege? O que a lógica ganha ao abraçar sua teoria? Tratamos dessas e outras questões ao longo deste texto.

Para tanto, na primeira seção, apresentamos as vertentes da lógica que a divide em duas correntes de pensamento nos quais estão situadas as contribuições de seus colaboradores, quais sejam, a lógica enquanto linguagem universal e a lógica enquanto cálculo, ou teoria dos modelos. Pretendemos situar Peirce um uma dessas tradições e a partir daí compreender alguns aspectos de sua teoria.

$\mathrm{Na}$ segunda seção, abordamos os eixos mais fundamentais do pensamento peirciano e sua originalidade diante da tradição. $O$ que apenas Peirce legou para 0 estudo da lógica e como proceder diante disso? Para responder a essa questão nos debruçaremos sobre as seis especificidades da teoria peirciana levantadas por Dipert e a forma como elas o tornam um pensador singular.

Por fim, na terceira seção, utilizamos como carro chefe o texto de Anellis, How Peircean was the "Fregean' Revolution" in Logic? Para argumentar que Peirce trabalhou, em certos pontos ainda mais cedo, as mesmas questões abordadas por Frege e Russell, compreendidas como responsáveis pela revolução da lógica no século XIX. Apresentamos os sete pontos que, segundo Heijenoort, foram as novidades trazidas pela tradição fregeana para a lógica e argumentamos a partir do texto de Anellis que todos os pontos foram também abordados por Peirce, embora não reconhecidos pela linha mainstream da lógica.

\section{Lógica enquanto linguagem como meio universal e como teoria dos modelos}

Se temos como intuito situar Peirce na tradição sobre os estudos da lógica, devemos primeiro reconhecer as vertentes na história da teoria lógica para então apontar o lugar do pensador em relação aos outros autores. Partindo de certos aspectos do trabalho de Peirce, pretendemos nessa seção mensurar seu pensamento enquanto pertencente à abordagem lógica da linguagem como meio universal (universal medium), ou da linguagem como cálculo, chamada ainda visão da linguagem segundo a teoria dos modelos (model-theoretical view of language) de acordo com a análise de Hintikka (1997).

Um adepto da universalidade da linguagem entende esta como um intermediário indispensável entre o sujeito e seu mundo. Nessa perspectiva a linguagem nos mantém como "prisioneiros", uma vez que não se pode dar um passo além dela para entender o modo como ela se relaciona com o mundo, isto é, não se 
pode expressar na linguagem sua relação semântica com o mundo sem cometer nonsense ou tautologia. Na perspectiva da universalidade da linguagem, portanto, a semântica é inefável, ou seja, não se pode descrever ou nomear sua natureza.

A concepção da linguagem enquanto cálculo implica a possibilidade de realizar todas as proezas que os universalistas insistem em dizer serem impossíveis, por exemplo, discutir a semântica da linguagem através da própria linguagem. Nessa perspectiva também podemos variar a interpretação de nossa linguagem, isto é, admitir outros modelos para suas proposições além do universo em questão. Essa forma de entender a linguagem torna-a um cálculo no qual pode-se atribuir livremente uma nova interpretação. No entanto, um defensor dessa abordagem não compreende a linguagem como um cálculo não interpretável, mas apenas que ela é reinterpretável como um cálculo o é. A linguagem aqui não é apenas uma ferramenta para inferências puramente formais.

Embora a tese da universalidade da linguagem possa parecer mais distante do que sua rival, ela é dominante no pensamento lógico inicial assim como nos princípios da filosofia da linguagem analítica. A visão universalista foi abraçada por Frege, o jovem Russell, Wittgenstein, o Círculo de Viena e, em certa medida, Quine. Do lado então chamado continental, a mesma crença na inefabilidade da verdade e outros conceitos semânticos é compartilhada por Heidegger, cuja influência tornou o princípio universalista a pedra angular das tradições hermenêutica e desconstrutivista. (HINTIKKA, 1997)

Contra tais abordagens filosóficas de enorme aceitação, a tradição da linguagem enquanto cálculo pode parecer insignificante na história da teoria da lógica (SLUGA, 1987). Essa tradição inclui como representantes Boole, Schöder e Löwenheim em seu início e ganha uma maior importância entre os lógicos através dos trabalhos de Tarski, Gödel e Carnap, embora tenha se tornado ainda menos influente entre os filósofos.

Diante da descrição de ambas as vertentes, Hintikka mantém como argumento a inserção de Peirce como membro integral da tradição da teoria dos modelos, ou seja, da linguagem enquanto cálculo. Segundo o autor, Peirce identificou o propósito sério da sua linguagem dos grafos dizendo que "o sistema não pretende servir como uma linguagem universal para matemáticos ou outros pensadores como aquele de Peano" (CP 4.424). Peirce ainda afirma "em minha álgebra e grafos, longe de ser tentado qualquer coisa desse tipo [resolver problemas por sistemas de expressões mais familiares], todo o esforço tendo sido como dissecar as operações de inferência em tantos passos distintos quanto possível" (CP 4.424).

Sobre o posicionamento peirciano dentro das perspectivas aqui expostas, Rodrigues (2017) aponta, divergindo de Hintikka e aliando-se a Anellis (2012) ${ }^{1}$, que Peirce é um membro de ambas as vertentes. O autor defende tal posicionamento em seu artigo Squaring the unknown: the generalization of logic according to G. Boole, A. De Morgan and C. S. Peirce do qual selecionamos o seguinte trecho: 1 O argumento de Anellis será abordado na terceira seção desse trabalho, mais especificamente no
item 3.6. 
Para Peirce, lógica pode ser entendida em um sentido estrito e em um amplo. O sentido estrito é a lógica formal, lógica escrita com uma linguagem matemática, a qual ele chama lógica exata, seguindo Schroeder. Mas, num sentido geral, lógica é uma ciência normativa, de um grau mais elevado e que não pode ser confundida com a lógica exata, porque se tata verdadeiramente da geral e quasinecessária doutrina dos signos, que Peirce, de modo idiossincrático, chama de Semiótica. Como tal, lógica não pode ser reduzida à matemática, embora sendo uma ciência menos geral, usa princípios e métodos matemáticos em suas investigações [...] Este, é claro, não se trata de um anti-logicismo ingênuo, mas uma concepção completamente original de objetivos e propósitos de diferentes atividades científicas. (RODRIGUES, 2017, p. 37)

A posição de Peirce implica a importância da tentativa de situá-lo na tradição da lógica, inclusive como denúncia à atenção quase exclusiva à teoria universalista da linguagem. Frente isso, podemos questionar a premissa de que Frege é o fundador da lógica contemporânea (HEIJENOORT, 1967). Por que não Peirce? Frege, de fato, foi o primeiro a publicar uma teoria sobre as partes básicas da lógica como a teoria da quantificação, cálculo de predicados e a lógica de primeira ordem. Porém, Peirce desenvolveu, junto de seus antecessores, uma lógica dos quantificadores completamente independente de Frege. Peirce não apenas descobriu de maneira independente a importância dos quantificadores e desenvolveu uma notação para eles, como também discutiu sua natureza de uma forma mais perceptiva. De modo geral, o montante de ferramentas lógicas encontradas em Peirce são muito mais ricas do que aquelas deixadas por Frege (HINTIKKA, 1997).

A vertente universalista de Frege ajudou no caráter impressionante de seu trabalho, inclusive por ser impulsionado por outros lógicos universalistas como Russell e Quine. Frege apresentou a lógica de primeira ordem como a parte central de uma linguagem universal relativamente simples a qual é calculada para capturar fielmente o mundo. Para Peirce, a mesma teoria da quantificação é apenas um tipo de sistema lógico entre tantos outros. Assim afirma o filósofo "o método universalmente válido é aquele da demonstração matemática; e esse é o único comumente evitado pelos lógicos como falacioso" (NEM 4:21).

\section{O lugar de Peirce na história da lógica}

Segundo Dipert (1995), o lugar de Peirce na história da lógica é difícil de descrever, embora o autor considere-o, mesmo assim, um Lógico que se equipara a Aristóteles, Boole e Frege. Para defender seu posicionamento, Dipert levanta três eixos, segundo os quais podemos considerar a lógica de Peirce hoje. Primeiro, podemos enxergá-lo como um descobridor, isto é, como primeiro na história da lógica a desenvolver certas ideias. Segundo, podemos nos interessar pelas influências históricas da lógica de Peirce e como ele contribuiu com grandes resultados na lógica matemática na primeira metade do século XX. Por fim, devemos notar o mérito das ideias distintas de Peirce na Lógica e sua utilidade. Para apresentar o posicionamento de Dipert sobre tal assunto, usaremos seu artigo Peirce's Underestimated Place in the History of Logic: a Response to Quine, da forma como se segue. 
Apenas para enunciar de modo breve, Peirce demonstrou originalidade em alguns pontos específicos em sua teoria ao, a) desenvolver uma teoria de uma lógica simbólica relacional; b) usar a expressão e conceito de "quantificação"; c) usar "índices" como variáveis, e de $\Pi$ e $\Sigma$ como quantificadores, ligando-os; d) o uso independente da notação "saturação" para descrever uma expressão predicada completa; e) desenvolver numerosas sugestões de formulações do tipo axiomáticas de teorias lógicas; e f) sugerir algo como uma percepção da expressiva capacidade de predicados lógicos proposicionais, de classes e monádicos, de um lado, e uma lógica de predicados de primeira ordem completa com múltiplas relações de quantificadores, do outro, assim como corrigir algumas notações e técnicas. Estes entre outros aspectos espalhados no corpus do texto peirciano.

O verdadeiro valor de Peirce, segundo Dipert, está no seu refinamento e visões idiossincráticas da filosofia da lógica e da matemática. Não é possível falar de "Peirce o lógico" sem necessariamente se chegar a Peirce enquanto filósofo e o meta-matemático. Dipert indica, para uma melhor apreciação do pensamento peirciano, algumas áreas do trabalho de lógica realizados por Peirce que continuam possuindo inestimado interesse e valor.

Primeiro, nota-se o expressivo poder das teorias algébricas. Peirce, em seus estudos, desenvolveu um cálculo sem operadores de combinar variáveis (variablebinding operators) tal como feito por Frege e Russell. Tal cálculo é capaz de combinar operadores proposicionais, de inclusão de classes e relacionais.

Segundo, compreender a lógica como parte da matemática. Diferentemente dos universalistas, Peirce compreende a lógica como parte da matemática e não o contrário, o que o livra de uma postura logicista. Matemática para Peirce é mais do que uma teoria semântica e rigorosa de diagramas e representações formais usadas pela racionalidade. A matemática inclui as regras de gramática e transformação das linguagens naturais. O princípio que configura tal expressão é o de que todos os pensamentos e formas de comunicação são diagramáticos e a questão é qual dos diagramas são mais "úteis"2 em um sentido mais robusto.

Terceiro, lógica como uma ciência normativa, não fundacional. A lógica para Peirce possui um propósito normativo: melhorar ou facilitar nossa habilidade de pensar, seja na matemática ou em outras áreas. Peirce concebia o sistema notacional como capaz de facilitar a compreensão e representação de pensamentos complexos. Apenas em Peirce podemos observar visões sofisticadas sobre diagramas, representações e ícones.

Quarto, a semântica. Peirce afirma que lógica proposicional, dos predicados e até meta-lógica podem ser tratadas formalmente pelo mesmo cálculo básico, utilizando sua "—<" e sua sugestão de que domínios de discurso podem ser compostos de itens incomuns, tais como possibilia, entidades abstratas e absurdas, ou até mesmo entidades não discretas, ou seja, um continuum. Peirce rejeitou os esforços reducionistas de seus predecessores e contemporâneos em tranformar a lógica proposicional em lógica de classes pelo truque semântico em declarar o universo de discurso como uma classe de tempos, instantes ou ocasiões. Ele viu a lógica proposicional, de classes e relacional como relações de meta-lógica, tais como as consequências lógicas, ilações, como espécies de um gênero diagramático mais geral.

\footnotetext{
2 Usamos aqui o termo "útil", mas prezamos as ressalvas necessárias ao Pragmaticismo.
} 
Quinto, a teoria peirciana dos conjuntos. Peirce considerou as visões de Cantor e Dedekind e discutiu questões fundacionais na teoria dos conjuntos de modo sofisticado. Sua discussão sobre as fundações filosóficas da teoria dos conjuntos é especialmente profunda e preenche lacunas deixadas por Cantor e seus sucessores, inclusive Frege. A questão filosófica substancial do que um conjunto é ou deveria ser tem sido pouco desenvolvida desde as observações de Cantor e muitas vezes, entendidas pelo autor como teológicas. Peirce propôs uma discussão sobre a natureza ontológica dos conjuntos, fomentando assim essa área de estudos.

Sexto, lógica e todo pensamento. Encontramos em Peirce, não apenas a qualidade normativa da lógica, mas também a concepção da lógica enquanto a maneira de como pensamentos sucedem uns aos outros. Peirce viu a lógica como o estudo do valor positivo no pensamento e, especialmente, em inferências de todos os tipos. Através de seu ensaio The Fixation of Belief, de 1870, a questão da inferência se tornou uma teoria do método científico e sua concepção enquanto abdução, dedução e indução.

\section{O sistema Peirciano e sua injusta ofuscação pelo sistema dos Universalistas}

Pretendemos nessa seção, de acordo com o artigo de Anellis (2012), How Peircean was the "Fregean' revoluiton" in Logic?, apresentar o modo como a teoria peirciana já abordava em seu sistema os sete pontos levantados por Heijenoort (1967) segundo os quais Frege, e mais tarde Russell, haviam mudado a história da lógica. Anellis nos apresenta, ponto-a-ponto, como Peirce já havia lidado com, e solucionado, as mesmas questões apresentadas pelos universalistas.

3.1 O cálculo proposicional de Peirce com uma definição vero-funcional (Truthfunctional definition) de conectivos, especialmente da condicional.

Em uma breve comparação entre as fórmulas de Peano/Russell, Peirce e as de Schöder, as diferenças encontradas são pura e simplesmente questões de notação, de acordo com a lógica proposicional.

No manuscrito On the Algebraic Principles of Formal Logic, escrito em 1879, o mesmo ano em que a Begriffsschrift de Frege vem à público, Peirce identificou a sua "garra" (claw ou hook), representada pelo símbolo "—<", como cópula de inclusão e definiu a implicação material ou inferência lógica, ilação, como " $1^{\circ}, A-<A$, qualquer que seja $A .2^{\circ}$, se $A-<B$, e $B-<C$, então $A-<C$ ".

Mais adiante, tornou-se possível distinguir proposições universais e particulares, afirmativas e negativas, de acordo com o esquema seguinte:
A. $\quad a \longrightarrow b$ Todo $A$ é $B$
(universal afirmativa)
E. $a<\bar{b}$ Nenhum $A$ é $B$
(universal negativa)
I. $\quad \breve{a} \longrightarrow b \quad \operatorname{Algum} A$ é $B$
(particular afirmativa)
O. $\breve{a} \longrightarrow \bar{b} \quad$ Algum $A$ não é $B$
(particular negativa) 
A partir dessas relações, Peirce ocupou-se da análise vero-funcional ${ }^{3}$ das proposições e das provas, assim como introduziu considerações vero-funcionais específicas, dizendo que, usando "v" como símbolo para verdadeiro e " $\mathrm{f}$ " para falso, as proposições " $\mathrm{f}-<a$ " e " $a-<\mathrm{v}$ " são verdadeiras, e um ou outro de " $v-<a$ " ou "a — < f" são verdadeiras, dependendo da verdade ou falsidade de $a$, e continuou a análise das propriedades vero-funcionais da "garra".

Utilizando seu novo arcabouço conceitual lógico, Peirce reescreve o silogismo de Aristóteles, do modo como exposto a seguir e em comparação com o modelo fregiano exposto em sua Begriffsschrift:

a) Peirce

$$
\begin{aligned}
\text { If } & x<y, \\
\text { and } & y<z \\
\text { then } & x<z .
\end{aligned}
$$

b) Frege

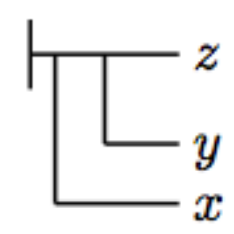

Outro passo significativo dos estudos da condicional desenvolvidos por Peirce foi a criação das tabelas de verdade que, diferentemente daquilo que prega a tradição, não foi pensada desenvolvida primeiramente por Post ou Wittgenstein, mas sim pelo pensador americano. Dada a fórmula $\{(\overline{(a-b)}-c)<d\}<e$, uma das linhas de sua tabela de verdade poderia ser traçada do seguinte modo:

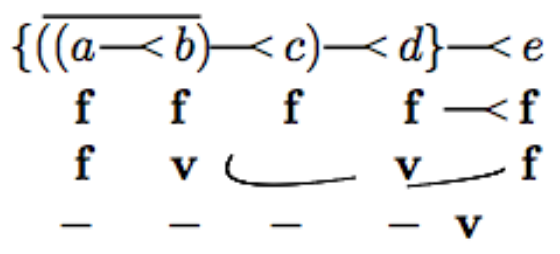

Em 1902, Peirce demonstrou a tabela (que podemos ler como a da bicondicional material) que segue para três termos, $x, y, z$, escrevendo $v$ para verdadeiro e f para falso, cujo formato é bastante semelhante aqueles utilizados na lógica contemporânea:

\begin{tabular}{c|c|c}
$x$ & $y$ & $z$ \\
\hline $\mathbf{v}$ & $\mathbf{v}$ & $\mathbf{v}$ \\
$\mathbf{v}$ & $\mathbf{f}$ & $\mathbf{f}$ \\
$\mathbf{f}$ & $\mathbf{v}$ & $\mathbf{f}$ \\
$\mathbf{f}$ & $\mathbf{f}$ & $\mathbf{v}$
\end{tabular}

3 Usamos aqui a tradução do termo Truth-functional conforme sugere J. Ferrater Mora no seu Dicionário de Filosofia, Edições Loyola, 2004. 
Na qual z é um termo derivado de uma operação lógica sobre os termos x e y. Peirce aplicou seu método tabular para vários conectivos, por exemplo a negação de X, comr - ‘, no seu Logic Notebook 1865-1909, e com V, F e L representava os valores de verdade verdadeiro, falso e indeterminado ou desconhecido, respectivamente, o qual chamou de limite (limit).

3.2 Decomposição de proposições em função e argumento ao invés de sujeito e predicado.

A lógica de Aristóteles, que se manteve até o século XIX como teoria acabada, chamada de lógica silogística, ou lógica dos termos, considerava suas proposições enquanto constituídas por uma sintaxe entre sujeito e predicado, ligados entre si por uma cópula. Peirce, em sua empreitada lógica, lidou com a Lógica de Aristóteles, assim como a de Boole e De Morgan e chegou a uma lógica formal que combina esses autores e vai além ao permitir que sua cópula de ilação mantenha, dependendo do contexto, termos do silogismo, classes e proposições e expanda esses, afim de desenvolver uma teoria da quantificação em sua lógica dos relativos.

Gilbert Ryle (1957), mesmo admitidamente sabendo que a ideia de relação foi proposta de maneira "respeitável" por De Morgan, atribuiu, de qualquer forma, a Russell, no The Principles of Mathematics, ao invés de Peirce, sua codificação. Ryle afirmou que as potencialidades do padrão relacional $x R y$, em oposição ao já sobrecarregado padrão $s-p$, foram logo muito bem apreciadas por filósofos que esperavam, por meio dele, ordenar todos os tipos de saber.

Mitchell definiu polinômios lógicos indexados (indexed logical polynomials), tais como " $l i, j$ ", como funções de uma classe de termos, nos quais para o polinômio lógico $F$ enquanto função de uma classe de termos $a, b, \ldots$, do universo de discurso $U, F 1$ é definido como "Todo $U$ é $F$ " e $F u$ é definido como "Algum $U$ é $F$ ". O que Mitchell produziu é um refinamento da notação que Peirce planejou para sua álgebra dos relativos possibilitando a distinção entre os termos dos polinômios pela indexação de termos, e adicionando o índice dos quantificadores variando nos termos dos polinômios. As melhorias de Mitchell foram imediatamente adotadas por Peirce e permitiram que o autor desenvolvesse uma teoria da quantificação de primeira ordem tão expressiva quanto a de Frege.

3.3 A teoria da quantificação de Peirce baseada em um sistema de axiomas e regras inferenciais

Os esforços de Peirce para desenvolver sua teoria da quantificação de primeira ordem publicada em 1883 em The Logic of Relatives teve início pelo menos antes de 1867 em seu texto On an Improvement in Boole's Calculus of Logic e adiante foi melhorada pelas inovações quanto as notações de Mitchell em On a New Algebra of Logic de 1883. Em 1885, Peirce publica On the Algebra of Logic: A Contribution to the Philosophy of Notation onde apresenta, além de uma teoria da 
quantificação de primeira ordem, também uma teoria da quantificação de segunda ordem.

A versão final da teoria de primeira ordem de Peirce usa índices para enumerar e distinguir os objetos considerados na parte booleana de uma equação tanto quanto índices de quantificadores, um conceito tirado de Mitchell. Peirce denotou os quantificadores existencial e universal por " $\Sigma_{i}$ " e " $\eta_{i}$ ", respectivamente, como somas e produtos lógicos, e variáveis individuais i, j, ..., são atribuídas para ambos quantificadores e predicados, isto é, para ambos quantificadores e para o termo distinto dos polinômios lógicos da parte booleana da equação. Escreveu, a partir disso, "li,j" para "i é o amante de j". "Todo mundo ama alguém" é escrito pelo sistema peirciano como " $\Pi_{\mathrm{i}} \Sigma_{\mathrm{j}} l_{\mathrm{l}, \mathrm{j}}$ ", isto é, "Todo mundo é o amante de alguém".

\subsection{A definição de Peirce de sequências infinitas e números naturais em termos de} noções lógicas (isto é, a logicização da matemática)

Frege desenvolveu sua teoria das sequências definidas em termos de noções lógicas na parte final de sua Begriffsschrift através da qual nos apresenta a relação ancestral e depois o próprio ancestral, o último exigido para garantir que as sequências chegadas sejam bem ordenadas. Com o ancestral próprio, finalmente ele é capaz de definir também a indução matemática.

Peirce, em On the Logic of Number, estabeleceu uma axiomatização da teoria dos números, começando pela definição de conjunto finito (finite set) para obter números naturais. Dado um conjunto $N$ e $R$ uma relação sobre $N$, com 1 um elemento de $\mathrm{N}$; com definições de mínimo, máximo e predecessor no tocante a $\mathrm{R}$ e $\mathrm{N}$ dados, os axiomas peircianos em terminologia moderna são:
a) $\quad \mathrm{N}$ é parcialmente ordenado por $\mathrm{R}$.
b) $\quad \mathrm{N}$ é conectado por $\mathrm{R}$.
c) N é fechado em relação aos predecessores.
d) 1 é o elemento mínimo de $\mathrm{N}$; $\mathrm{N}$ não possui máximo.
e) Indução matemática vale para (holds for) $\mathrm{N}$.

As únicas diferenças significantes entre a teoria da axiomatização do número de Richard Dedekind (1888) e a de Peirce é que Dedekind partiu de conjuntos infinitos ao invés de conjuntos finitos para definir números naturais e que Dedekind é explicitamente e especificamente preocupado com o contínuo de números reais, isto é, com conjuntos infinitos. Peirce rejeitou o contínuo real em favor do infinitesimal Leibniziano. Além disso, Peirce rejeitou conjuntos transfinitos mantendo a posição de que Cantor e Dedekind eram incapazes de apoiar logicamente a construção do infinito real, e que somente o infinito potencial poderia ser estabelecido logicamente.

Para Peirce o objeto por trás da axiomatização do sistema de número naturais foi afirmado em On the Logic of Number como estabelecendo que proposições elementares relativas ao número são apresentadas pelas demonstrações usuais. De modo geral, Peirce queria aqui estabelecer que o sistema de números naturais pode ser desenvolvido axiomaticamnete por métodos dedutivos aplicando sua lógica das 
relações o sistema de números naturais pode ser construído por esse meio sobre as bases de algumas definições lógicas.

\subsection{A representação e clarificação de Peirce do conceito de sistema formal}

Geraldine Brady (2000) afirmou que Peirce falhou em fornecer um sistema lógico formal no mesmo sentido que Frege. A motivação para a criação de tal sistema não está presente no trabalho de Peirce. De fato, no sistema peirciano não há um conjunto de axiomas dos quais poderia derivar toda a lógica, muito menos a matemática. Ao invés disso, tem-se um experimento contínuo no desenvolvimento da base de uma lógica que responde a propósitos específicos e possui como objetivo final a criação de um cálculo que sirva como ferramenta para a concepção mais ampla da lógica enquanto teoria dos signos.

Portanto, Peirce não apresentou em um trabalho completo e coerente um sistema formal universalmente abrangente, ao invés disso, produziu uma série de sistemas formais, ainda que apresentados de maneira informal. Esses sistemas trabalham não com um universo de discurso universal, tal como o de Frege, mas com universos de discurso específicos.

3.6 A lógica e Semiótica de Peirce, tornando possível, e dando, um uso da lógica para investigações filosóficas (especialmente para a filosofia da linguagem)

O entendimento de Van Heijenoort acerca da concepção de aplicação da teoria da teoria lógica de Frege para a investigação filosófica e, em particular, para a filosofia da linguagem, pode ser visto de duas formas. Primeiro, O programa logicista de Frege foi entendido como a peça central e dizia respeito à articulação das ciências, incluindo a matemática, desenvolvida dentro da estrutura da teoria lógica; Segundo, a teoria de Frege é mais amplamente compreendida como tentativa de desenvolver uma teoria lógica como linguagem universal.

Van Heijenoort analisa a história da lógica de acordo com as duas vertentes que abordamos na primeira seção deste texto, quais sejam, a lógica enquanto linguagem universal e enquanto cálculo. É dele essas definições.

Diferentemente do que afirma Hintikka, tal como exposto na primeira seção, Anellis compreende o lugar de Peirce diante das vertentes lançadas por Heijenoort de modo peculiar. Partindo da definição de Peirce expressa em The Nature of Mathematics de que a "Lógica é a ciência que examina signos, verifica o que é essencial para ser signo e descreve suas variedades fundamentalmente diferentes, indaga sobre as condições gerais de sua verdade e as declara com precisão formal, e investiga a lei do desenvolvimento do pensamento, enuncia com precisão e enumera seus modos de trabalho fundamentalmente diferentes", enquanto o que ele chamou de "crítica" é aquela parte da lógica que diz respeito à dedução, e é, portanto, um cálculo. Anellis afirma, diante disso, que a lógica de Peirce é tanto um cálculo, enquanto crítica, e uma linguagem, enquanto teoria semiótica. Um cálculo no sentido estrito e uma linguagem num sentido amplo. 
3.7 A distinção de Peirce entre proposições singulares, tal como "Sócrates é mortal", de proposições universais como "Todos os Gregos são mortais"

A questão em distinguir proposições singulares de universais foi uma das motivações iniciais no empreendimento de Peirce em seu trabalho On an Improvement in Boole's Calculus of Logic, o qual tem como objetivo aprimorar a lógica algébrica de Boole através do desenvolvimento de uma teoria da quantificação que introduziria um uso mais perspicaz e eficiente dos quantificadores universal e existencial na álgebra de Boole e permitiria, da mesma forma, uma distinção clara entre proposições singulares e universais. Esse trabalho veio a total fruição em 1885 com a notação Mitchell/Peirce para fórmulas quantificadas para ambas regras indiciais (indexed) e inferenciais (inference).

Contudo, proposições singulares, especialmente aquelas em que ocorrem descrições definidas em vez de nomes próprios, também foram denominadas "proposições russellianas" assim chamadas por sua designação por Russell em termos do quantificador I (iota) ou operador I empregando um I invertido para ser lido como "o indivíduo X"; assim, por exemplo, (IX) (X), e nós temos, por exemplo, "Machado de Assis = (Ix) (x escreveu Dom Casmurro)".

No Principia Mathematica, Whitehead e Russell escreveram " $\varphi$ ! $x$ " para a função de primeira ordem de um indivíduo, isto é, para qualquer valor da variável que envolve apenas indivíduos; Desse modo, por exemplo, poderíamos escrever " $\mu$ !" (Sócrates) como "Sócrates é um homem". Na seção "Descrições" do Principia, o operador I substitui a notação " $\varphi$ ! $x$ " para singulares com " $(\mathrm{IX}) \Phi(\mathrm{X})$ " para que possa lidar com descrições definidas assim como nomes de indivíduos.

\section{Considerações Finais}

Diante do exposto, pode-se observar a injusta exclusão de Peirce na história da lógica e, consequentemente, a ofuscação de sua importância para a história do pensamento. Peirce merece estar ao lado dos grandes lógicos adotados pela tradição como Aristóteles, Frege, Russell entre outros.

Embora nossa empreitada tenha sido comparar os conceitos e a elaboração destes entre Peirce e os Universalistas, sobretudo Frege, não mantemos como pretensão afirmar que a teoria de Peirce é "melhor", ou que tenha sido pioneira. Reconhecemos que o objetivo de cada um desses pensadores é diferente, isto é, enquanto Frege está preocupado com a questão da justificação, Peirce empreende esforços para desenvolver a questão heurística da lógica. Diante desses posicionamentos opostos, a comparação entre tais teorias de modo a enaltecer uma ou outra é pobre e filosoficamente desnecessária.

Reafirmamos, contudo, que Peirce é um pensador fundamental para a história do pensamento e sua teoria sistêmica é bastante rica e inovadora em diversas áreas de estudo. Fica claro, portanto, o modo como a Lógica apenas ganha ao voltar sua atenção para o posicionamento peirciano e o desenvolvimento de suas ideias. 


\section{REFERÊNCIAS:}

ANELLIS, I. H. How Peircean was the "Fregean revolution" in logic? In: Logicheskie issledovaniya, no. 18, 2012. 239-272.

BRADY, G. From Peirce to Skolem: A Neglected Chapter in the History of Logic. Amsterdam/New York: North-Holland, 2000.

DEDEKIND, R. Was sind und was sollen die Zahlen? Braunschweig: F. Vieweg, 1888.

DIPERT, R. R. Peirce's Underestimated Place in the History of Logic: a Response to Quine. In: Peirce and contemporary Thought: Philosophical Inquiries. KETNER, K. L. (editor). New York: Fordham University Press, 1995. 32-58.

FREGE, G. Begriffsschrift, eine der arithmetischen nachgebildete Formelsprache des reinen Denkens. Halle: Verlag von Louis Nebert, 1879.

HEIJENOORT, J. Logic as calculus and logic as language, Synth'ese 17, 324330, 1967.

HINTIKKA, J. The Place of C. S. Peirce in the History of Logical Theory. In: The Rule of Reason: The Philophy of Charles Sanders Peirce. BRUNNING, J; FOSTER, P. (editors). Toronto: University of Toronto Press, 1997. 13-33.

PEIRCE, C. S. On an improvement in Boole's calculus of logic (Paper read on 12 March 1867), Proceedings of the American Academy of Arts and Sciences 7:250261, 1868.

. On the algebra of logic: a contribution to the philosophy of notation, American Journal of Mathematics 7: 180-202, 1885.

1881.

. On the logic of number, American Journal of Mathematics 4:85-95,

. The logic of relatives. In: Studies in Logic by Members of the Johns Hopkins University. Boston: Little, Brown \& Co., 1883. 187-203.

PEIRCE, C. S. (C. Hartshorne and P. Weiss, editors), Collected Papers of Charles Sanders Peirce, Vol. IV: The Simplest Mathematics. Cambridge, Mass., Harvard University Press, 1933; 2nd ed., 1961.

. Collected Papers of Charles Sanders Peirce, vol. V: Pragmatism and Pragmaticism. Cambridge, Mass.: Harvard University Press, 1934.

PEIRCE, C. S. (C. J. W. Kloesel, editor), Writings of Charles S. Peirce: A Chronological Edition, vol. 4: 1879-1884. Bloomington/Indianapolis: Indiana University Press, 1989.

Writings of Charles S. Peirce: A Chronological Edition, vol. 5: 18841886. Bloomington/Indianapolis: Indiana University Press, 1993.

POST, E. L. Introduction to a General Theory of Elementary Propositions, Ph.D. thesis, Columbia University. Abstract presented in Bul letin of the American Mathematical Society 26:437; abstract of a paper presented at the 24 April meeting of the American Mathematical Society, 1920.

Introduction to a general theory of elementary propositions. American Journal of Mathematics 43:169-173, 1921. 
QUINE, W. N. Peirce's Logic. In: Peirce and contemporary Thought: Philosophical Inquiries. KETNER, K. L. (editor). New York: Fordham University Press, 1995. 23-31. RODRIGUES, C. T. Squaring the unknown: the generalization of logic according to G. Boole, A. De Morgan and C. S. Peirce. In: South America Journal of Logic. Vol. 3. N. 2. p 1-67. 2017.

RUSSELL, B. Principles of Mathematics. Cambridge: Cambridge University Press, 1903.

RYLE, G. Introduction. In: A. J. Ayer, et. al., The Revolution in Philosophy. London: Macmillan \& Co.; New York: St. Martin's Press, 1957. Pp. 1-12.

SLUGA, H. Frege against the Booleans. In: Notre Dame Journal of Formal Logic 28: 1987. 80-93.

WHITEHEAD, A. N. and RUSSELL, B. Principia Mathematica, 3 vols. Cambridge: Cambridge University Press, 1910-13. 